\title{
Armazenamento, estabilidade oxidativa e caracterização do biodiesel metílico de soja
}

Storage, oxidative stability and characterization of soybean methyl biodiesel

\author{
A. U. Antunes Júnior ${ }^{1 *}$; A. S. Silva ${ }^{2}$; M. W. N. C. Carvalho ${ }^{1}$; K. R. O. Pereira ${ }^{3}$ \\ ${ }^{1}$ Unidade Acadêmica de Engenharia Química/Laboratório de Catálise, Adsorção e Biocombustíveis, Universidade \\ Federal de Campina Grande, 58429-140, Campina Grande-Paraíba, Brasil. \\ ${ }^{2}$ Unidade Acadêmica de Tecnologia de Alimentos, Universidade Federal de Campina Grande, 58840-000, Pombal- \\ Paraíba, Brasil.
}

${ }^{3}$ Unidade Acadêmica de Engenharia de Materiais/Laboratório de Síntese de Materiais Cerâmicos, Universidade Federal de Campina Grande, 58429-140, Campina Grande-Paraíba, Brasil.

*adaliciouzeda@hotmail.com

(Recebido em 23 de outubro de 2015; aceito em 02 de dezembro de 2016)

O biodiesel tem sido destaque no mercado mundial de combustíveis por ser proveniente de material renovável e ser menos poluente quando comparado aos combustíveis derivados do petróleo. Sendo de origem animal ou vegetal, o biodiesel apresenta, também, as mesmas rotas de degradação que estas matérias-primas. Este trabalho foi desenvolvido com o objetivo de avaliar as propriedades químicas e físicas, a estabilidade oxidativa e armazenamento de biodiesel metílico de soja aditivado com e sem antioxidante. A influência dos parâmetros tempo e incidência de luz na qualidade do biodiesel foram avaliados a partir de caracterizações do índice de acidez, índice de peróxido, viscosidade cinemática e massa específica. Os resultados sugerem o melhor desempenho à estabilidade oxidativa ao biodiesel aditivado com $1000 \mu \mathrm{g} \cdot \mathrm{mL}^{-1}$ de BHA, bem como as amostras que não foram submetidas à incidência de luz.

Palavras-chave: BHA (Butil Hidroxianisol), transesterificação, degradação.

Biodiesel has been featured on the world market for fuels come from renewable material and be less polluting when compared to petroleum fuels. Being of animal or vegetable, biodiesel also presents the same routes degradation of these materials. This work was carried out with the objective to evaluate the chemical and physical properties in oxidative stability, storage and termoxidative process of soybean methyl biodiesel additive with and without antioxidant. The influence of time and incidence of light parameters on the quality of biodiesel were evaluated from characterizations of acid index, peroxide index, kinematic viscosity and specific mass. The results suggest the best performance to oxidative stability biodiesel additive with $1000 \mu \mathrm{g} \cdot \mathrm{mL}^{-1}$ BHA, as well as samples that were not subject to the incidence of light.

Keywords: BHA (Butyl Hydroxyanisol), transesterification, degradation.

\section{INTRODUÇÃO}

As energias provenientes de fontes renováveis são uma alternativa promissora frente às energias oriundas de fontes fósseis por permitirem um fornecimento de energia sustentável, com menor impacto ambiental e uma redução na dependência de combustíveis fósseis [1].

Neste contexto, ganha importância a discussão do papel dos biocombustíveis como alternativa para a atual matriz energética mundial. Dentre estes, o biodiesel tem ganhado destaque como energia renovável. O biodiesel é uma importante fonte de energia, por ser biodegradável e ambientalmente menos poluente em relação à emissão de gases [2; 3; 4]. Devido às imposições ambientais e limitações de recursos de combustíveis fósseis, o biodiesel tem se mostrado uma boa alternativa para minimizar ambos os problemas.

As razões que corroboram o interesse pelo biodiesel, investindo-o de uma importância estratégica frente aos derivados do petróleo, são devidas às vantajosas características que este biocombustível apresenta, como: ser renovável e biodegradável; proporcionar diversificação da matriz energética; ter menor emissão de gases poluentes; pode ser obtido a partir de várias fontes 
renováveis; ser perfeitamente miscível e físico-quimicamente semelhante ao óleo diesel de origem mineral $[5 ; 6 ; 7]$.

No Brasil, a soja é a oleaginosa mais utilizada para a produção de biodiesel, responsável por $70 \%$ a $80 \%$ da produção [8]. Nesse contexto, o óleo de soja se caracteriza por ser a principal matéria-prima utilizada na produção de biodiesel e por classificar o Brasil como o segundo maior produtor de biodiesel derivado de óleo de soja. O Brasil além de ser o segundo maior produtor e processador mundial de soja em grão, é o segundo exportador mundial de soja em farelo e o terceiro maior exportador de óleo [9].

O óleo de soja, matéria-prima utilizada neste projeto para a síntese do biodiesel, é tipicamente caracterizado pela presença dos ácidos graxos do tipo linoléico e oléico [10]. A presença de teor de ácidos graxos insaturados torna este óleo bastante suscetível à oxidação, característica que é inevitavelmente repassada aos ésteres metílicos ou etílicos (biodiesel) obtidos a partir desta matéria-prima [11].

A oxidação é a alteração mais importante em óleos e uma das principais causas de deterioração de produtos a base de óleo vegetal. Este processo corresponde a uma série complexa de reações que afeta profundamente a qualidade de óleos e de seus derivados $[12 ; 13 ; 14]$.

Dunn [15] e Pullen e Saeed [16] relatam que o biodiesel apresenta o mesmo perfil de ácidos graxos da fonte lipídica original, e devido ao fato de muitos óleos vegetais (soja, milho, canola, algodão, etc.) apresentarem significante quantidade de ácidos graxos com duplas ligações, a estabilidade do biodiesel à oxidação tem-se tornado uma crescente preocupação, especialmente em se tratando de armazenamento deste biocombustível em longo prazo.

Os grupamentos ésteres, formado entre a carboxila do ácido graxo e a hidroxila do glicerol ou de outros álcoois, pertencem à classe de relativa reatividade das moléculas lipídicas, hidrolisandose a ácidos graxos livres na maioria das vezes. Os outros sítios reativos da molécula lipídica são as duplas ligações presentes nas cadeias hidrocarbonadas de um número de ácidos graxos, essas ligações são sensíveis às reações de oxidação, as quais são provocadas, na maioria dos casos, pelo oxigênio e por outros agentes oxidantes [17].

Os produtos (primários ou secundários) oriundos da decomposição oxidativa do biodiesel são os principais fatores a afetar a qualidade deste biocombustível, já que alguns podem afetar propriedades como a viscosidade, a densidade, o ponto de fulgor, e o índice de cetano [18].

Como todo processo de degradação existente em materiais orgânicos, a dos lipídeos é afetada por vários fatores que, diretamente e indiretamente, catalisam a sua decomposição química. Fatores intrínsecos, como o grau de insaturação dos lipídeos e o teor de compostos pós e antioxidantes, e fatores extrínsecos, como temperatura, oxigênio, luminosidade, íons metálicos de dupla valência, merecem destaque quando o assunto abordado for o armazenamento deste biocombustível $[18 ; 19 ; 20 ; 21]$.

Com a finalidade de inibir ou retardar a oxidação do biodiesel são empregados compostos químicos conhecidos como antioxidantes. A avaliação do uso de antioxidantes em biodiesel é profundamente difundida na literatura e os mais utilizados como inibidores de oxidação de biodiesel são: o butil hidroxianisol (BHA), o butil hidroxytolueno (BHT), butil hidroquinona (TBHQ), galato de propila (GP), dentre outros [18;22; 23; 24; 25; 26; 27].

Assim, o presente estudo teve por objetivo avaliar a estabilidade oxidativa do biodiesel metílico de óleo de soja, com e sem antioxidante, submetido a dois processos de oxidação acelerada. Adicionalmente, buscou-se estudar o comportamento ao longo do armazenamento e analisar a influência da ação da luminosidade ambiente sobre a estabilidade oxidativa do biodiesel. Com o intuito de analisar das propriedades físicas e químicas, foram aplicadas as caracterizações do índice de acidez, índice de peróxido, viscosidade cinemática e massa específica.

\section{MATERIAL E MÉTODOS}

O presente estudo foi realizado no Laboratório de Catálise, Adsorção e Biocombustíveis da Unidade Acadêmica de Engenharia Química, sediado na Universidade Federal de Campina 
Grande. Como matéria-prima utilizou-se o óleo de soja adquirido em mercado local do município de Campina Grande-PB.

Para a síntese do biodiesel utilizou-se o processo de transesterificação. Maior reatividade na produção de biodiesel é observada para álcoois de cadeia curta, assim, foi utilizado o metanol. Na reação utilizou-se uma razão molar de óleo: álcool de $1: 6$, temperatura de $60{ }^{\circ} \mathrm{C}$ e $1 \%$ de hidróxido de potássio (em relação à massa de óleo) como catalisador. A reação foi conduzida por 3 horas. Após esta etapa o biodiesel bruto foi disposto em funil de decantação tipo pêra para posterior separação do biodiesel e glicerol.

A purificação constou de lavagem prévia do biodiesel com solução de ácido clorídrico $(0,01$ $\%$ ) aquecida a $50{ }^{\circ} \mathrm{C}$ e posterior lavagem com água destilada até que a água descartada na lavagem ficasse com $\mathrm{pH}$ neutro. $\mathrm{O}$ biodiesel lavado permaneceu, ainda, no funil de decantação para separação da fase aquosa e em seguida foi realizada a secagem.

A secagem do biodiesel foi realizada em um sistema composto por agitador magnético com aquecimento, frasco kitassato para o biodiesel e sílica gel. A pressão foi reduzida em $550 \mathrm{mmHg}$ e a temperatura ficou em $75{ }^{\circ} \mathrm{C}$ por 45 minutos.

O biodiesel aditivado com três níveis de concentração de BHA (200, 600 e $\left.1000 \mu \mathrm{g} \cdot \mathrm{mL}^{-1}\right)$ e sem antioxidante foi avaliado durante o Método de Estufa de Schaal (MES), processo termoxidativo e armazenamento do biodiesel.

\subsection{Método de Estufa de Schaal}

O MES foi realizado de acordo com a metodologia descrita por Orthoefer e List [28]. O teste de estabilidade consistiu na exposição das amostras em estufa com circulação de ar a $60{ }^{\circ} \mathrm{C}$ por um período de 7 dias. Utilizou-se um total de $300 \mathrm{~mL}$ de biodiesel, os quais foram distribuídos aleatoriamente em vinte e quatro frascos de vidro âmbar (seis para cada nível do biodiesel aditivado e seis para o biodiesel não aditivado). Para avaliação química e física do material, a cada 3 dias, retirou-se uma alíquota total de $12 \mathrm{~mL}$ dos diferentes frascos para cada nível de concentração do aditivo.

\subsection{Processo termoxidativo}

O processo termoxidativo foi realizado de acordo com a metodologia descrita por Dunn [15], que consistiu em submeter as amostras à oxidação por meio de aquecimento e injeção de ar atmosférico.

O sistema utilizado no processo termoxidativo consistiu de um balão de fundo chato, condensador espiralado, agitador magnético com aquecimento e sistema de injeção de ar atmosférico acoplado a dois recipientes contendo sílica gel, o qual teve como objetivo manter o ar o mais seco possível (medido com termo-higrômetro digital Instrutherm TH-200).

Foram utilizados $75 \mathrm{~mL}$ de amostra. A temperatura do processo de oxidação foi de $110{ }^{\circ} \mathrm{C}$ (aferidos com termômetro de mercúrio). O fluxo de ar ambiente seco foi injetado a $10 \mathrm{~L}^{-\mathrm{h}^{-1}}$, correspondendo a um tempo total do processo de 12 horas. Foram coletadas amostras no início e no fim do processo para as devidas avaliações.

\subsection{Armazenamento do biodiesel}

Utilizou-se para o armazenamento em temperatura ambiente cerca de $700 \mathrm{~mL}$ do biodiesel sem antioxidante e aditivado com três níveis de concentração de BHA (200, 600 e $\left.1000 \mu \mathrm{g} \cdot \mathrm{mL}^{-1}\right)$. As amostras foram distribuídas em frascos de vidro transparentes com capacidade de $1000 \mathrm{~mL}$ e uma outra amostra base ( $0 \mu \mathrm{g} \cdot \mathrm{mL}^{-1}$ de antioxidante) foi acondicionada também em frasco âmbar, onde se avaliou o efeito da luminosidade no biodiesel não aditivado, sendo esta denominada SL.

O armazenamento foi realizado por um período de 90 dias sendo as amostras monitoradas por meio de determinações químicas (índice de acidez e índice de peróxido) e físicas (massa específica e viscosidade cinemática). 


\subsection{Avaliações químicas e físicas}

Os testes de estabilidade oxidativa, o armazenamento do biodiesel e o efeito da adição do antioxidante BHA foram avaliados quanto ao índice acidez, ao índice de peróxido, à viscosidade cinemática e à massa específica.

Determinou-se o índice de acidez mediante a metodologia descrita por Zenebon et al. [30], que se baseou na titulação da amostra com uma solução de hidróxido de sódio a 0,01 eq.L $L^{-1}$, e teve como solvente uma solução de 1:1 de éter etílico e álcool etílico neutralizados.

$\mathrm{O}$ índice de peróxido foi obtido mediante a metodologia descrita por Zenebon et al. [30], o qual procedeu com a titulação da amostra com uma solução de tiossulfato de sódio a 0,01 eq..$^{-1}$ na presença de amido, solução de ácido acético e clorofórmio (3:2) e iodeto de potássio.

A massa específica foi determinada em densímetro digital Anton Paar modelo DMA 35N à temperatura de $20^{\circ} \mathrm{C}$ (obtido em banho termoestático) de acordo com a metodologia descrita em norma ASTM [31].

A viscosidade cinemática foi determinada a $40{ }^{\circ} \mathrm{C}$ de acordo com a metodologia descrita na norma ASTM [32] em tubo viscosímetro Cannon-Fenske para líquidos transparentes.

\section{RESULTADOS E DISCUSSÃO}

\subsection{Método de Estufa de Schaal}

Na Figura 1 encontram-se apresentados os resultados referentes à avaliação da oxidação das amostras a partir do índice de peróxido obtido no Método de Estufa de Schaal (MES). O uso do antioxidante BHA está apresentado nas concentrações de 200, 600 e 1000 ppm, enquanto que o 0 ppm refere-se ao experimento sem uso do BHA

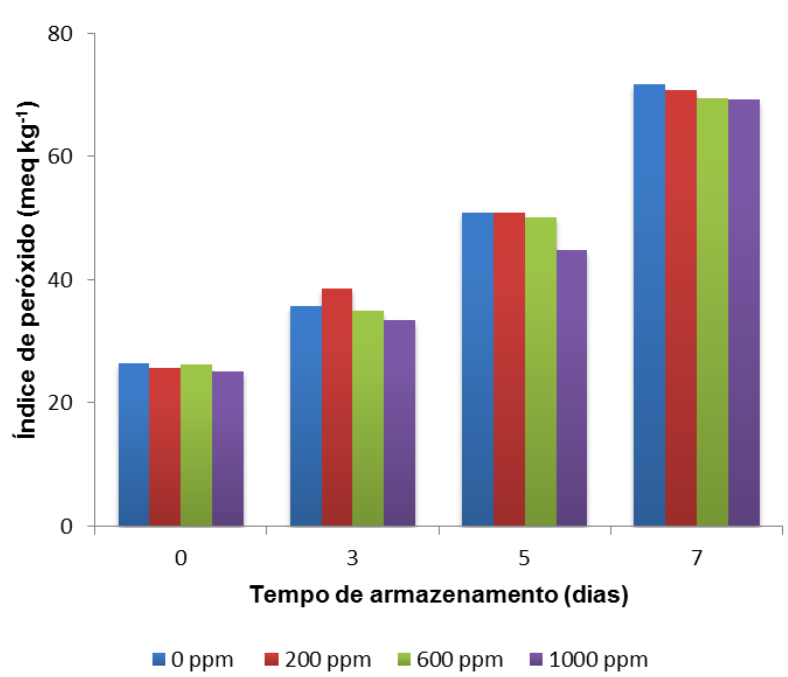

Figura 1: Índice de peróxido do biodiesel obtidos no MES em função do tempo de armazenamento.

Mediante análise destes resultados, verificou-se que o biodiesel demonstra suscetibilidade à oxidação ao longo do processo oxidativo. Além disso, foi constatado que o biodiesel aditivado com $1000 \mu \mathrm{g} \cdot \mathrm{mL}^{-1}$ do antioxidante apresentou menor valor para esta resposta avaliada em todos os intervalos de tempo.

Ao término do processo, observou-se que os valores obtidos para o índice de peróxido aumentaram drasticamente em relação aos valores iniciais. Tal fato constata que quanto maior o tempo de armazenamento no Método de Estufa de Schaal, e consequentemente, maior influência do efeito térmico nas amostras, menor será a resistência à oxidação do biodiesel. Resultados semelhantes quanto às elevações do índice de peróxido são observados ao longo do Método de Estufa de Schaal na literatura $[33 ; 34 ; 35]$. 


\subsection{Processo Termoxidativo}

A viscosidade e a massa específica são parâmetros importantes na qualidade do biodiesel e podem ser diretamente relacionadas com a pureza do produto obtido, assumindo que não ocorram possíveis interações entre o éster metílico e os glicerídeos. De acordo com Filippis et al. [36] e Issariyakul e Dalai [10] fatores como reação incompleta, a presença de mono, di e triglicerídeos ou a presença de glicerina, devido à purificação ineficiente, promovem mudanças na viscosidade e na massa específica.

De acordo com Pullen e Saeed [16] as viscosidades elevadas em uma amostra de biodiesel podem ser conferidas à presença de triacilglicerídeos, principais constituintes de óleos vegetais, responsáveis pelo alto grau de polimerização que dificulta o uso deste combustível in natura em motores a diesel.

Na Figura 2a encontram-se apresentados os resultados da massa específica analisados para as amostras de biodiesel submetidas ao processo termoxidativo de 12 horas.

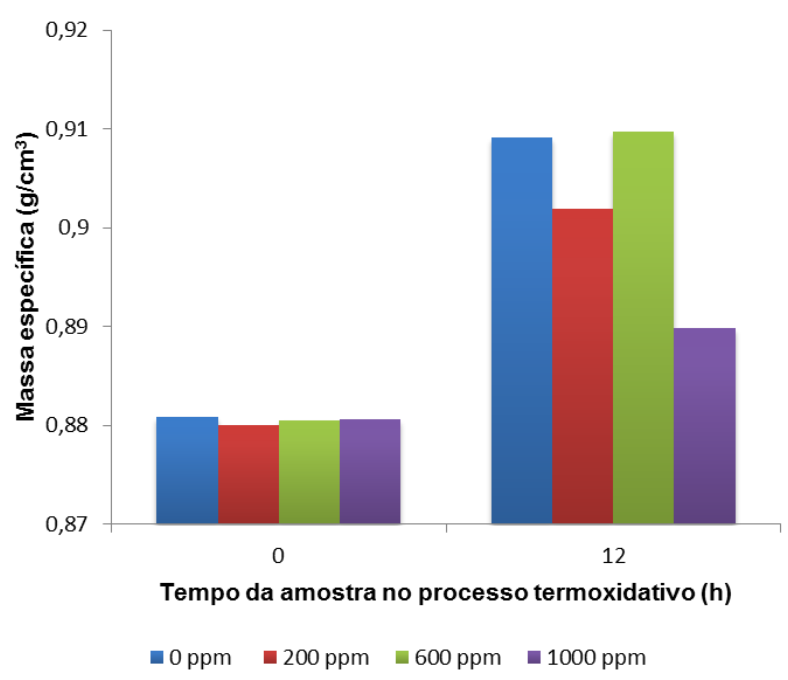

(a)

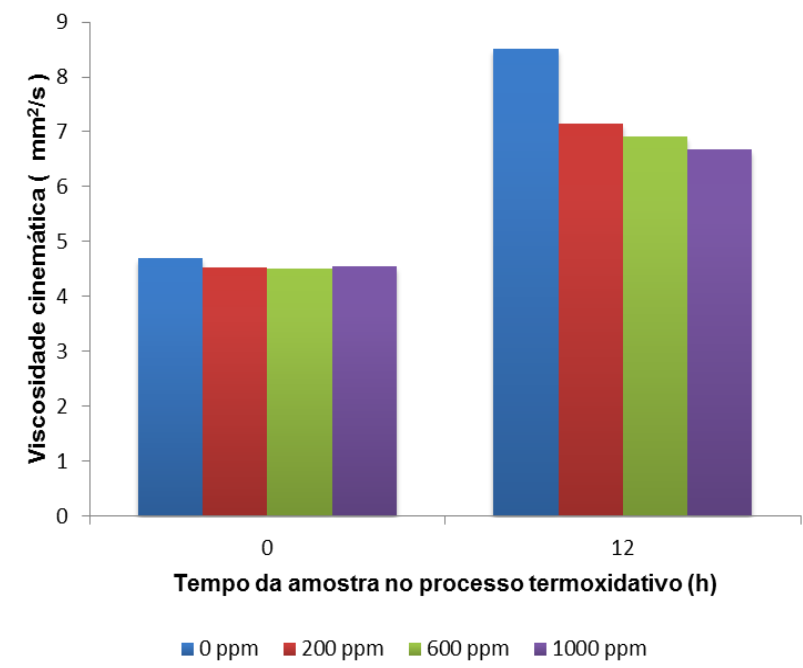

(b)

Figura 2: Massa específica (a) e viscosidade cinemática (b) obtidas no processo termoxidativo em função do tempo de armazenamento.

Verifica-se aumento da massa específica e que apenas a amostra aditivada com $1000 \mu \mathrm{g} \cdot \mathrm{mL}^{-1}$ do BHA ao término no processo termoxidativo encontra-se dentro dos padrões estabelecidos para este parâmetro $\left(0,85 \mathrm{~g} / \mathrm{cm}^{3}\right.$ até $\left.0,90 \mathrm{~g} / \mathrm{cm}^{3}\right)$ pela Resolução ANP nº5/ 2014 [37]. Além disso, 
observou-se que a amostra com $600 \mu \mathrm{g} \cdot \mathrm{mL}^{-1}$ não promoveu a redução do valor da massa específica e apresentou comportamento bem semelhante à amostra base, tal fato pode ser atribuído à possível polimerização dos triacilglicerídeos durante o teste.

No que diz respeito à viscosidade cinemática (Figura $2 b$ ), constatou-se elevação deste valor ao longo do processo termoxidativo e menor viscosidade cinemática presente no biodiesel de óleo de soja com $1000 \mu \mathrm{g} \cdot \mathrm{mL}^{-1}$ do BHA. Este parâmetro permaneceu dentro do exigido por lei ANP [37], que é de 3 a $6 \mathrm{~mm}^{2} \cdot \mathrm{s}^{-1}$ nas amostras iniciais, porém ao término do processo, as amostras apresentaram valores que estão em desacordo com o exigido pela resolução. A elevação da viscosidade cinemática possivelmente foi causada devido ao elevado grau de polimerização dos triacilglicerídeos, citado anteriormente, que aumenta a resistência do fluido ao escoamento, podendo gerar problemas de circulação e injeção do combustível nos motores diesel, assim como observado nos estudos de Parente [38] e Pullen e Saeed [16].

Nas Figuras $3 \mathrm{a}$ e $3 \mathrm{~b}$ encontram-se apresentados os resultados referentes ao índice de acidez e peróxido obtidos no processo termoxidativo do biodiesel metílico de óleo de soja.

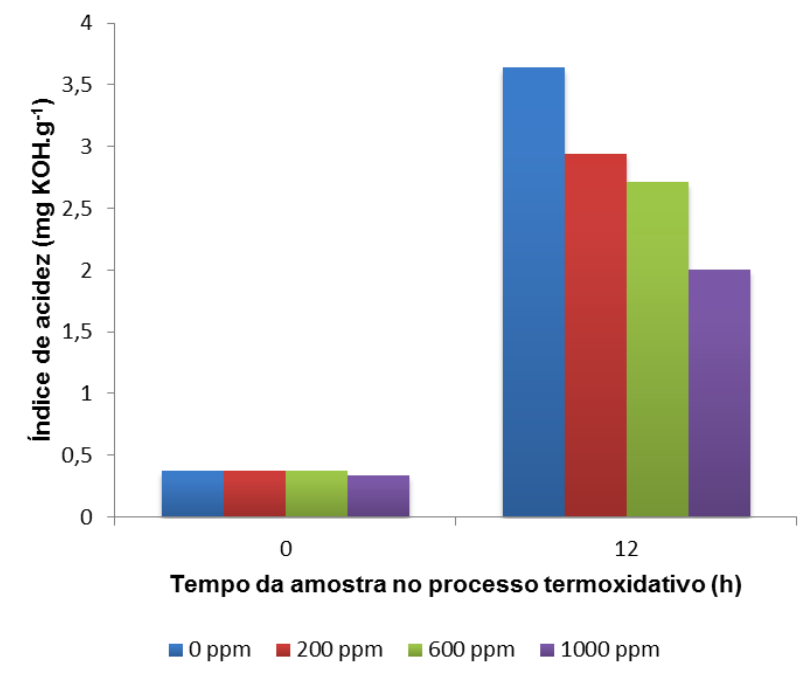

(a)

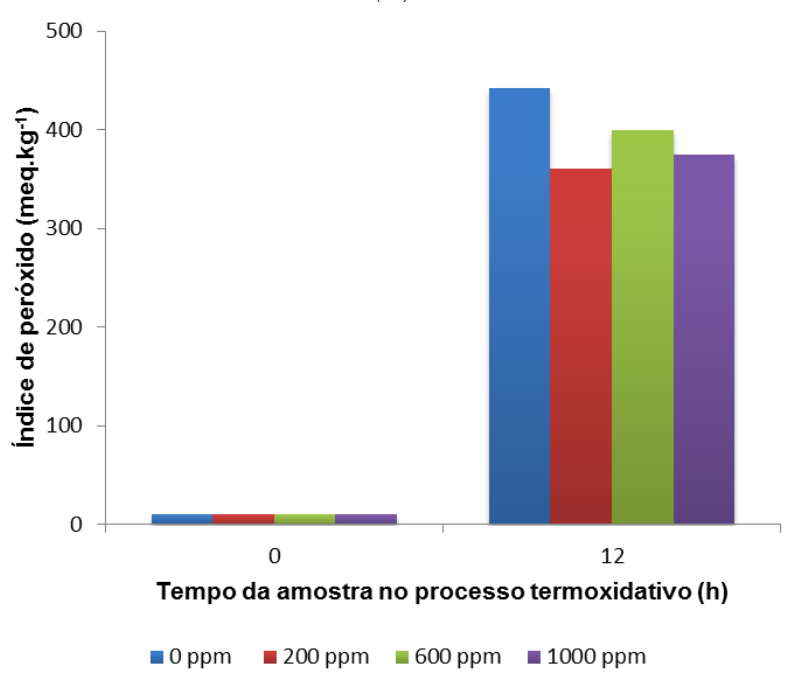

(b)

Figura 3: Índice de acidez (a) e índice de peróxido (b) obtidos no processo termoxidativo em função do tempo de armazenamento.

A acidez de um óleo e do produto final é de extrema importância para se evitar problemas com relação ao processo reacional, como consumo excessivo do catalisador, ocorrência de reações paralelas assim como também a não ocorrência da reação. Dantas et al. [39] afirmam que os índices de acidez e ácidos graxos livres influenciam na hidrólise e oxidação do biodiesel quando 
em valores acima dos limites máximos permissíveis das normas estabelecidas pela ANP [37]. Além disso, a acidez elevada pode catalisar reações intermoleculares dos triacilglicerídeos ao mesmo tempo em que afeta a estabilidade térmica do combustível na câmara de combustão, além de apresentar ação corrosiva sobre os componentes metálicos no motor.

Mediante análise dos resultados presentes na Figura 3a, constatou-se que para o índice de acidez, a amostra aditivada com $1000 \mu \mathrm{g} \cdot \mathrm{mL}^{-1}$ de BHA apresentou maior estabilidade oxidativa, seguida das amostras com 600 e $200 \mu \mathrm{g} \cdot \mathrm{mL}^{-1}$. Consoante com estudos desenvolvidos por Ferrari et al. [40] e Dantas [39], o índice de acidez para a amostra do biodiesel pura inicial esteve abaixo de $0,55 \mathrm{mg}$ de $\mathrm{KOH} . \mathrm{kg}^{-1}$ apresentando-se dentro do estabelecido pela ANP [37].

De acordo com Pullen e Saeed [16], índice de peróxido representa uma das formas para avaliar o grau de oxidação de fontes lipídicas. Fatores como temperatura, enzimas, luz e íons metálicos podem influenciar a formação de radicais livres. O radical livre em contato com oxigênio forma um peróxido que, em reação com outra molécula oxidável, induz a formação de hidroperóxido e outro radical livre.

Ao avaliar os resultados apresentados na Figura 3(b), verificou-se que o processo termoxidativo promoveu um aumento muito considerável do índice de peróxido ao comparar os valores iniciais e após as 12 horas do teste e, consequentemente, proporcionou a oxidação do biodiesel. Em estudos de Borsato et al. [24], Dinkov et al. [42] e Taghvaei et al. [43], ao aplicarem diferentes antioxidantes, verificou-se que o efeito da temperatura e injeção de ar atmosférico no processo termoxidativo reduziu o efeito dos inibidores e promoveu aumento do índice de peróxido de material oleaginoso.

Observa-se que as amostras aditivadas com 200 e $1000 \mu \mathrm{g} \cdot \mathrm{mL}^{-1}$ do antioxidante apresentaram maior estabilidade oxidativa. Adicionalmente, verifica-se que a amostra com $600 \mu \mathrm{g} \cdot \mathrm{mL}^{-1}$ apresentou menor estabilidade oxidativa quando comparado à amostra com nível de concentração de $200 \mu \mathrm{g} . \mathrm{mL}^{-1}$ de BHA. Uma possível razão desta oscilação encontra-se no fato da volatilidade dos peróxidos durante o processo realizado, assim como observado nos estudos de Jorge [44] referente à estabilidade oxidativa de óleos vegetais e óleos de frituras.

\subsection{Armazenamento do biodiesel}

Na Tabela 1 encontram-se os resultados referentes aos resultados de índice de acidez, índice de peróxido, viscosidade cinemática e massa específica em relação ao biodiesel aditivado no início do armazenamento.

Tabela 1: Caracterizações químicas e físicas do biodiesel metílico de soja.

\begin{tabular}{|c|c|c|c|c|c|}
\hline \multirow[t]{2}{*}{ Determinações } & \multirow{2}{*}{$\begin{array}{c}\text { Amostra } \\
\text { base }\end{array}$} & \multicolumn{3}{|c|}{$\begin{array}{c}\text { Concentrações de BHA } \\
\left(\mu \mathrm{g} . \mathrm{mL}^{-1}\right)\end{array}$} & \multirow[t]{2}{*}{ ANP* } \\
\hline & & 200 & 600 & 1000 & \\
\hline Índice de acidez (mg.KOH g ${ }^{-1}$ ) & 0,545 & 0,598 & 0,624 & 0,607 & $<0,50$ \\
\hline Índice de peróxido (meq.kg-11) & 26,57 & 25,72 & 26,36 & 25,23 & - \\
\hline Viscosidade cinemática $\left(\mathrm{mm}^{2} \cdot \mathrm{s}^{-1}\right)$ & 4,49 & 4,47 & 4,55 & 4,55 & 3 a 6 \\
\hline Massa específica $\left({\left.\mathrm{g} . \mathrm{cm}^{-3}\right)}^{-3}\right.$ & 0,8805 & 0,8806 & 0,8816 & 0,8805 & 0,85 a 0,90 \\
\hline
\end{tabular}

* Agência Nacional de Petróleo, Gás Natural e Biocombustíveis.

A influência do uso de antioxidantes na alteração das avaliações químicas e físicas na estabilidade oxidativa do biodiesel foi também estudada por Ferrari e Oliveira [45], em que se percebeu que o uso do BHA apresentou resultados intermediários quando comparados aos antioxidantes TBHQ e BHT na suscetibilidade à oxidação.

Conforme análise realizada, observou-se que houve elevação do índice de acidez à medida que se elevaram as concentrações do antioxidante, estando o biodiesel fora das normas estabelecidas pela ANP [37]. 
Em relação aos valores do índice de peróxido, verifica-se que os valores se mantiveram bem próximos independente da concentração do antioxidante. Mesmo comportamento foi observado para os parâmetros de viscosidade cinemática e massa específica, estando ambos acetáveis para os limites das normas estabelecidas pela ANP [37].

Estudos desenvolvidos por Agarwal e Khurana [20], Serrano et al. [26], Rizwanul Fattah et al. [13] relatam que a estabilidade à oxidação diminui quando o biodiesel é armazenado por longos períodos de tempo. A oxidação durante o armazenamento do biodiesel leva ao aumento da viscosidade, o aumento do valor de peróxido, aumento do número de ácido, e diminuição período de indução. Assim, é de fundamental importância o acompanhamento das propriedades físicas e químicas do biodiesel durante o período de armazenamento.

Nas Figuras 4a e 4b encontram-se apresentados os resultados referentes à massa específica e viscosidade cinemática, obtidos ao longo do armazenamento do biodiesel de óleo de soja.

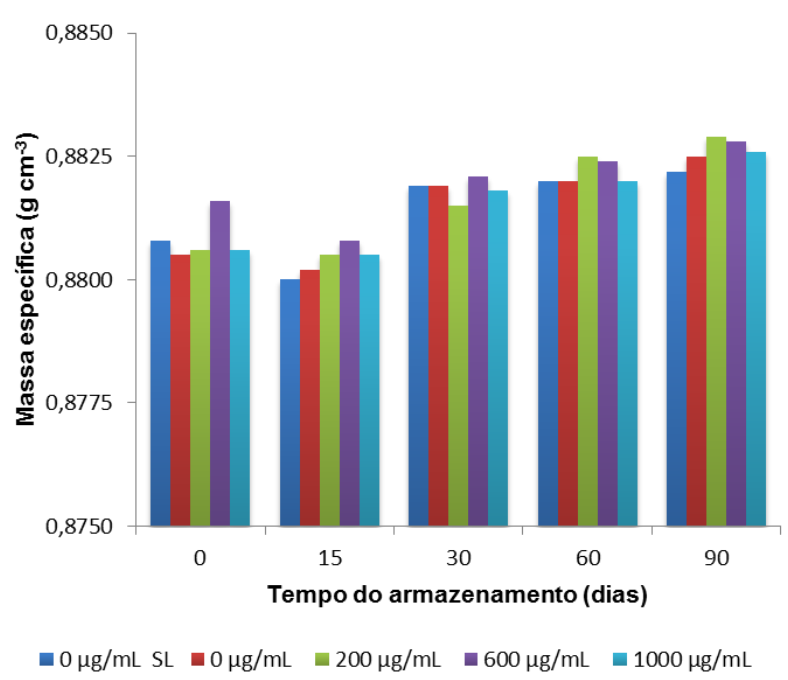

(a)

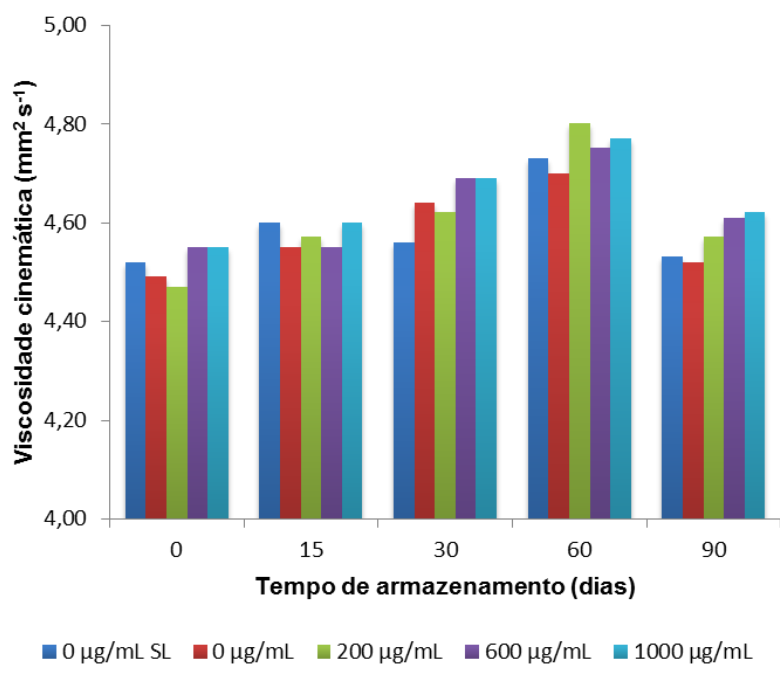

(b)

Figura 4: Massa específica (a) e viscosidade cinemática (b) em função do tempo de armazenamento.

A análise dos resultados referentes a massa específica (Figura 4a) demonstrou que mesmo após 90 dias de armazenamento, as amostras apresentaram-se conformidade com a Resolução ANP $\mathrm{n}^{\circ} 45 / 2014$ [37] (com valores entre $0,85 \mathrm{~g} / \mathrm{cm}^{3}$ e $0,90 \mathrm{~g} / \mathrm{cm}^{3}$ ). Verifica-se ainda, a elevação deste valor à medida que transcorre o tempo de armazenamento do biodiesel. Resultados semelhantes foram encontrados em estudos desenvolvidos por Rizwanul Fattah et al. [13].

No que diz respeito à viscosidade cinemática (Figura 4b), contatou-se elevação deste valor ao longo dos 60 dias do armazenamento e um decréscimo ao final dos 90 dias. Tal fato pode ser um 
indicativo que a viscosidade do biodiesel não é diretamente proporcional ao tempo durante longos períodos de armazenamento, proporcionando uma redução da resistência ao escoamento após o período de 60 dias. Entretanto, este parâmetro permaneceu em conformidade com os valores de 3 a $6 \mathrm{~mm}^{2} / \mathrm{s}$ estabelecidos pela Resolução ANP n ${ }^{\circ} 45 / 2014$ [37].

A influência da luminosidade não apresentou impacto considerável nas modificações das propriedades físicas (viscosidade cinemática e massa específica) avaliadas nas amostras de biodiesel. As pequenas oscilações nos parâmetros de viscosidade cinemática e massa específica observadas ao longo do armazenamento do biodiesel foram também observadas em estudos desenvolvidos por Joshi et al. [12] e Sousa et al. [46].

Nas Figuras 5 a e 5 b encontram-se apresentados os resultados referentes às avaliações químicas do índice de acidez e índice de peróxido.

Ao avaliar os resultados apresentados na Figura 5a, observou-se que após 90 dias de armazenamento do biodiesel, a amostra com $1000 \mu \mathrm{g} \cdot \mathrm{mL}^{-1}$ de BHA apresentou menor índice de acidez quando comparada às demais amostras aditivadas, e resultado semelhante para o biodiesel sem antioxidante e com baixa influência da luminosidade. Tal fato indica que a influência da foto-oxidação proporciona alterações no valor do índice de acidez.

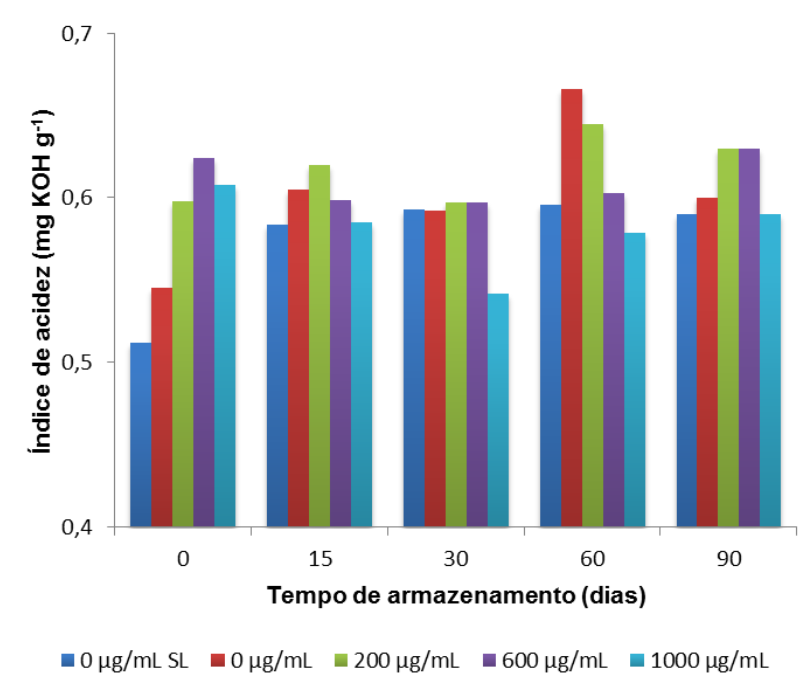

(a)

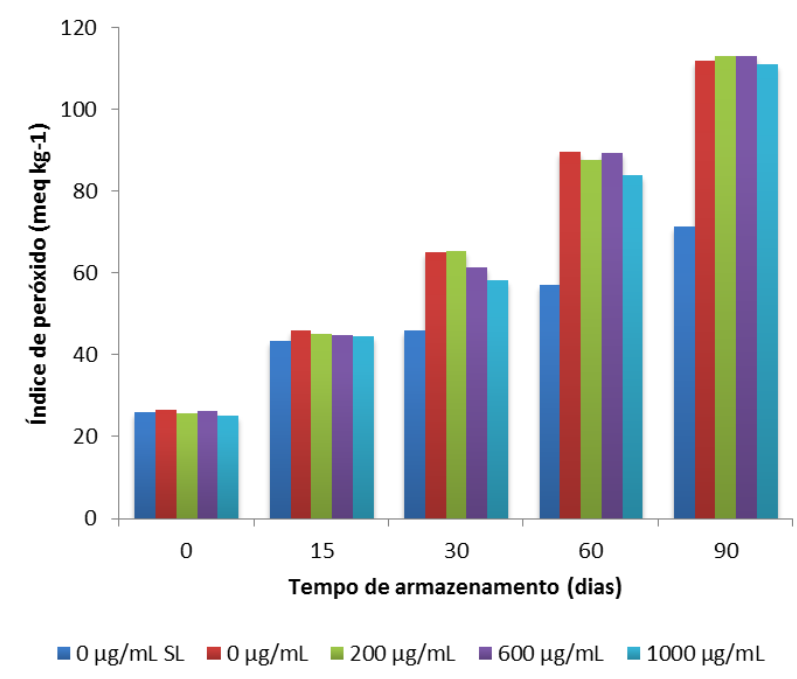

(b)

Figura 5: Índice de acidez (a) e índice de peróxido (b) em função do tempo de armazenamento.

Na Figura 5b constata-se a elevação do índice de peróxido, tornando o biodiesel mais suscetível à oxidação ao longo do armazenamento. Em relação às amostras aditivadas, verificou- 
se que até 60 dias de armazenamento, o biodiesel com nível de concentração de $1000 \mu \mathrm{g} \cdot \mathrm{mL}^{-1}$ apresentou melhor desempenho, no entanto, ao término dos 90 dias, percebeu-se comportamento bem semelhante para todas as amostras em relação à estabilidade oxidativa.

A amostra sem concentração do antioxidante BHA e com baixa influência da luminosidade apresentou melhor estabilidade oxidativa devido a menor degradação de peróxido durante o armazenamento e apresentou valores bem abaixo dos obtidos para as amostras aditivadas. Estudos da literatura [20] comprovaram que a exposição à luminosidade é uma condição adversa para o armazenamento do biodiesel e que promove uma redução drástica da estabilidade oxidativa do material oleaginoso.

A vulnerabilidade oxidativa do biodiesel à exposição da luminosidade por ação do efeito fotooxidativo explica o motivo pelo qual o biodiesel armazenado em um recipiente âmbar obteve melhor desempenho no índice de peróxido quando comparado às demais amostras aditivadas com o antioxidante BHA.

\section{CONCLUSÃO}

No MES, a maior estabilidade oxidativa foi conferida ao biodiesel aditivado com $1000 \mu \mathrm{g} . \mathrm{mL}^{-1}$ do BHA. Além disso, observou-se que quanto maior foi o tempo de armazenamento acelerado sob influência direta da temperatura, maior foi a suscetibilidade à oxidação do biodiesel.

A influência da injeção de ar atmosférico e elevação da temperatura, conforme observado no processo termoxidativo, é capaz de alterar drasticamente as propriedades físicas e químicas do biocombustível. Após o teste, verificou-se que o melhor desempenho foi conferido à amostra com maior concentração de antioxidante.

No que se refere ao armazenamento do biocombustível, observou-se que o tempo promoveu mudanças mais pronunciadas no índice de peróxido e índice de acidez, conferindo menor estabilidade oxidativa do biodiesel após os 90 dias de armazenamento. Além disso, dentre as amostras aditivadas, melhores resultados foram obtidos para o maior nível do antioxidante.

Adicionalmente, ao analisar o armazenamento do biocombustível, a amostra com baixa influência do efeito da luminosidade obteve melhor estabilidade oxidativa em relação ao índice de peróxido e desempenho semelhante ao biodiesel aditivado com $1000 \mu \mathrm{g} \cdot \mathrm{mL}^{-1}$ do BHA para as demais avaliações.

\section{AGRADECIMENTOS}

Ao CNPq pelo auxílio financeiro e ao Laboratório de Caracterização de Materiais/UAEMa/UFCG.

\section{REFERÊNCIAS BIBLIOGRÁFICAS}

1. Peterson CL, Feldman R, Khorus R. Batch type transesterification process for winter rape oil. Applied Engineering in Agriculture, 1991;7(6):711-716, doi: 10.13031/2013.26291.

2. Linares N, Silvestre-Albero AM, Serrano E, Silvestre-Albero J, García-Martiínez J. Mesoporous materials for clean energy technologies. Chemical Society Reviews. 2014 Apr;43:7681-7717, doi: $10.1039 / \mathrm{c} 3 \mathrm{cs} 60435 \mathrm{~g}$.

3. Serres JDS, Soares D, Corazza ML, Krieger N, Mitchell DA. Liquid-liquid equilibrium data and thermodynamic modeling for systems related to the production of ethyl esters of fatty acids from soybean soapstock acid oil. Fuel. 2015 May;147(1):147-154.

4. Hoekman SK, Broch A, Robbins C, Ceniceros E, Natarajan M. Review of biodiesel composition, properties, and specifications. Renewable and Sustainable Energy Reviews. 2012 Jan;16(1):143-169.

5. Gaio LM. Preparação, Caracterização e Uso de Dolomita Mineral como Catalisador Heterogêneo na Produção de Biodiesel via Reação de Transesterificação de Óleo de Fritura [dissertação], Brasília (DF): Universidade de Brasília, 2014.

6. Pukale DD, Maddikeri GL, Gogate PR, Pandit AB, Pratap AP. Ultrasound Assisted Transesterification of Waste Cooking Oil Using Heterogeneous Solid Catalyst. Ultrasonics Sonochemistry. 2014 Jan;22:278-286, doi:10.1016/j.ultsonch.2014.05.020. 
7. Dall'Oglio EL, Sousa Júnior PT, Oliveira PTJ, Vasconcelos LG, Parizotto A, Kuhnen CA. Use of Heterogeneous Catalysts in Methylic Biodiesel Production Induced by Microwave Irradiation. Química Nova. 2014 Fev;37(3):411-417, 2014.

8. Ching WH, Rodrigues CW. Cartilha biodiesel. SEBRAE: São Paulo. 2010.

9. Brasil, Ministério de Minas e Energia. Anuário estatístico brasileiro do petróleo, gás natural e biocombustíveis. Agência Nacional de Petróleo, Gás Natural e Biocombustíveis. 2014.

10. Issariyakul T, Dalai AK. Biodiesel from vegetable oils. Renewable and Sustainable Energy Reviews. 2014 Jan;31:446-471, doi: 10.1016/j.rser.2013.11.001.

11. Van Gerpen JH, Hammond EG, Monyen A. Determining the influence of contaminants on biodiesel properties. Society of Automotive Engineers Paper. International Spring Fuels \& Lubricants Meeting \& Exposition. 1997; doi:10.4271/971685.

12. Joshi G, Lamba BY, Rawat DS, Mallick S, Murthy KSR. Evaluation of Additive Effects on Oxidation Stability of Jatropha Curcas Biodiesel Blends with Conventional Diesel Sold at Retail Outlets. Industrial \& Engineering Chemistry Research. 2013 May;52(22):7586-7592, doi: 10.1021/ie400801h.

13. Rizwanul Fattah IM, Masjuki HH, Kalam MA, Masum BM. Effect of synthetic antioxidants on storage stability of Calophyllum inophyllum biodiesel. Materials Research Innovations. 2014;18(6):90-94.

14. Ali OM, Mamat R, Faizal CKM. Review of the effects of additives on biodiesel properties, performance, and emission features. Journal of Renewable and Sustainable Energy. 2013;5:1-15, doi: $10.1063 / 1.4792846$

15. Dunn RO. Effect of antioxidants on the oxidative stability of methyl soyate (biodiesel). Fuel Processing Technology. 2005 Jun;86(10):1071-1085, doi:10.1016/j.fuproc.2004.11.003.

16. Pullen J, Saeed K. An overview of biodiesel oxidation stability. Renewable and Sustainable Energy Reviews. 2012 Aug;16:5924-5950, doi:10.1016/j.rser.2012.06.024.

17. Oetterer M, Reginato-D'arce MAB, Spoto MHF. Fundamentos de Ciência e Tecnologia de Alimentos. Barueri: Editora Manole; 2004. 612 p.

18. Jakeria MR, Fazal MA, Haseeb ASMA. Influence of different factors on the stability of biodiesel: A review. Renewable and Sustainable Energy Reviews. 2014 Oct;30:154-163, doi: 10.1016/j.rser.2013.09.024.

19. Park JY, Kim DK, Lee JP, Park SC, Kim YJ, Lee JS. Blending effects of biodiesels on oxidation stability and low temperature flow properties. Bioresource Technology. 2008 Mar;99(5):1196-1203, doi: 10.1016/j.biortech.2007.02.017.

20. Agarwal AK, Khurana D. Long-term storage oxidation stability of Karanja biodiesel with the use of antioxidants. Fuel Processing Technology. 2013 Sep;106:447-452, doi: 10.1016/j.fuproc.2012.09.011.

21. Vieira FS, Pasquini C. Determination of the oxidative stability of biodiesel using near infrared emission spectroscopy. Fuel. 2014 Oct;117:1004-1009, doi: 10.1016/j.fuel.2013.10.027.

22. Goulart LA, Teixeira ARL, Ramalho DA, Terezo AJ, Castilho M. Development of an analytical method for the determination of tert-butylhydroquinone in soybean biodiesel. Fuel. 2014 Jul;115:126-131, doi: 10.1016/j.fuel.2013.06.050.

23. Medeiros ML, Cordeiro AMMT, Queiroz N, Soledade LEB, Souza AL, Souza AG. Efficient Antioxidant Formulations for Use in Biodiesel. Energy Fuels. 2014 Dec;28:1074-1080, doi: 10.1021/ef402009e.

24. Borsato D, Maia ECR, Dall’Antonia LH, Silva HC, Pereira JL. Cinética da oxidação de biodiesel de óleo de soja em mistura com TBHQ: determinação do tempo de estocagem. Quim Nova. 2012 Jan;35(4):733-737.

25. Caramit RP, Andrade AGF, Souza JBG, Araujo TA, Viana LH, Trindade MAG, Ferreira VS. A new voltammetric method for the simultaneous determination of the antioxidants TBHQ and BHA in biodiesel using multi-walled carbon nanotube screen-printed electrodes. Fuel. 2013 Jun;105:306-313, doi: 10.1016/j.fuel.2012.06.062.

26. Serrano M, Martínez M, Aracil J. Long term storage stability of biodiesel: Influence of feedstock, commercial additives and purification step. Fuel Processing Technology. 2013 Jun;116:135-141, doi: 10.1016/j.fuproc.2013.05.011.

27. Supriyono, Sulistyo H, Almeida MF, Dias JM. Influence of synthetic antioxidants on the oxidation stability of biodiesel produced from acid raw Jatropha curcas oil. Fuel Processing Technology. 2015 Jan;132:133-138, doi: 10.1016/j.fuproc.2014.12.003.

28. Orthoefer FT, List GR. Trait-Modified Oils in Foods. Wiley-Blackwell; 2015.

29. Dunn RO. Effect of oxidation under accelerated conditions on fuel properties of methyl soyate. Journal of the American Oil Chemists' Society. 2002;79(9):915-920.

30. Zenebon O, Pascuet NS, Tiglea P. Métodos físico-químicos para análise de alimentos. São Paulo: Instituto Adolfo Lutz; 2008. 596-598 p. 
31. American Society for Testing and Materials (ASTM). ASTM D445- Standard Test Method for Kinematic Viscosity of Transparent and Opaque Liquids (and calculation of Dynamic Viscosity). West Conshoocken, 2006.

32. American Society for Testing and Materials (ASTM). ASTM D4052- Standard Density and Relative Density of Liquids by Digital Density Meter. West Conshouhocken, 2006.

33. Borsato D, Cini JRM, Silva HC, Coppo RL, Angilelli KG, Moreira I, Maia ECR. Oxidation kinetics of biodiesel from soybean mixed with synthetic antioxidants BHA, BHT and TBHQ: Determination of activation energy. Fuel Processing Technology. 2014 Jul;127:111-116, doi: 10.1016/j.fuproc.2014.05.033.

34. Gruczynska E, Przybylski R, Aladedunye F. Performance of structured lipids incorporating selected phenolic and ascorbic acids. Food Chemistry. 2015 Oct;173:778-783, doi: 10.1016/j.foodchem.2014.10.122.

35. Aladedunye F, Niehaus K, Bednarz H, Thiyam-Hollander U, Fehling E, Matthaus B. Enzymatic lipophilization of phenolic extract from rowanberry (Sorbus aucuparia) and evaluation of antioxidative activity in edible oil. LWT - Food Science and Technology. 2015 Aug;60:56-62, doi: 10.1016/j.lwt.2014.08.008.

36. Filippis P, Giavarini C, Scarsella M, Sorrentino M. Transesterification processes for vegetable oils: A simple control method of methyl esters content. Journal of the American Oil Chemists Society. 1995;72:1399, 1995.

37. ANP. Agência Nacional do Petróleo, Gás Natural e Biocombustíveis [internet]. Resolução ANP n ${ }^{45 /}$ 2014. [acesso em 14 de outubro de 2015] Disponível em: http://nxt.anp.gov.br/NXT/gateway.dll/leg/resolucoes_anp/2014/agosto/ranp\%2045\%20$\%$ 202014.xml?fn=document-frameset.htm $\$ \mathrm{f}=$ templates $\$ 3.0$.

38. Parente EJS. Biodiesel: uma aventura tecnológica num país engraçado. Fortaleza: Tebio; 2003. 66 p.

39. Dantas HJ, Candeia RA, Conceição MM, Silva MCD, Santos IMG, Souza AG. Caracterização FísicoQuímica e Estudo Térmico de Biodiesel Etílico de Algodão. $1^{\circ}$ Congresso da Rede Brasileira de Tecnologia de Biodiesel; 2006 Ago 31 - Set 01; Brasília (DF); v. 1, p. 231-235.

40. Ferrari RA, Oliveira VS, Scabio A. Biodiesel de soja: taxa de conversão em ésteres etílicos, caracterização físico química e consumo em geradores de energia. Química Nova. 2005 Nov;28(1):1923.

41. Pullen J, Saeed K. Experimental study of the factors affecting the oxidation stability of biodiesel FAME fuels. Fuel Processing Technology. 2014 May;125:223-235, doi: 10.1016/j.fuproc.2014.03.032.

42. Dinkov RK, Stratiev DS, Shishkova IK, Ivanov SK, Tsaneva TT, Mitkova M, Skumov M. Assessment of shelf life of Bulgarian industrial FAME by the use of modified ASTM D2274 as accelerated oxidation method. Fuel Processing Technology. 2015 Nov;130:245-251, doi: 10.1016/j.fuproc.2014.10.016.

43. Taghvaei M, Jafari SM, Assadpoor E, Nowrouzieh S, Alishah O. Optimization of microwave-assisted extraction of cottonseed oil and evaluation of its oxidative stability and physicochemical properties. Food Chemistry. 2014 Mar;160:90-97, doi: 10.1016/j.foodchem.2014.03.064.

44. Jorge N. Estudo do comportamento do óleo de girassol e do efeito do dimetil polisiloxano em termoxidação e frituras. [tese]. Campinas (SP): Universidade Estadual de Campinas; 2009.

45. Ferrari RA, Oliveira VS. Avaliação da estabilidade oxidativa de biodiesel de óleo de girassol com antioxidantes. Química Nova. 2009 Dez;32(1):106-111.

46. Sousa LS, Moura CVR, Oliveira JE, Moura EM. Use of natural antioxidants in soybean biodiesel. Fuel. 2014 Oct;134:420-428, doi: 10.1016/j.fuel.2014.06.007. 